\title{
Difficulties in the diagnosis of periapical translucencies and in the classification of cemento-osseous dysplasia
}

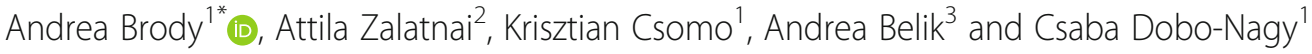

\begin{abstract}
Background: Cemento-osseous dysplasia is a benign fibro-osseous lesion of the tooth-bearing region of the jaws with a periodontal ligament origin. It appears predominantly in Black and Asian middle-aged females. Its importance is that it could mimic a periapical lesion in the early, translucent stage.

Case presentation: In this report a rare case of familial cemento-osseous dysplasia is presented: a 50-years old Caucasian woman with labial paraesthesia and radiological translucency around the roots of the mandibular incisors and the first molar teeth. The lesion around the first molar was diagnosed as periapical granuloma and a root canal treatment was carried out. The diagnosis of florid cemento-osseous dysplasia and the treatment plan based on two- and three-dimensional radiographic examinations were certified histologically after surgical removal of the lesion. We screened the family members - including the patient's mother, daughter and son - and identified a periapical version of cemento-osseous dysplasia in the daughter. Our case highlights the difficulties of differential diagnosis of cemento-osseous dysplasia and other periapical pathologies. The inconsistencies in the present classification of cemento-osseous dysplasia are also discussed with a proposal for a different classification based on new aspects that would be very helpful in setting up a correct treatment plan.
\end{abstract}

\section{Conclusion:}

- Differentiation of endodontic and non-endodontic origin of radiolucency and distinguishing it from anatomical landmarks by appropriate clinical evaluation and using vitality testing can give an opportunity to prevent unnecessary endodontic treatment.

- The current categories of cemento-osseous dysplasia classification do not cover the early stage of a hereditary florid form of cemento-osseous dysplasia.

- Instead of anatomical location of the lesion, clinical and genetic features may be recommended as parameters of cemento-osseous dysplasia classification.

Keywords: Cemento-osseous dysplasia, Classification, Differential diagnosis, Familial form of COD, Florid, Misdiagnosis, Periapical translucencies

\footnotetext{
*Correspondence: brody.andrea@gmail.com

'Department of Oral Diagnostics, Faculty of Dentistry, Semmelweis

University, Szentkirályi u. 47, Budapest 1088, Hungary

Full list of author information is available at the end of the article
}

(c) The Author(s). 2019 Open Access This article is distributed under the terms of the Creative Commons Attribution 4.0 International License (http://creativecommons.org/licenses/by/4.0/), which permits unrestricted use, distribution, and reproduction in any medium, provided you give appropriate credit to the original author(s) and the source, provide a link to the Creative Commons license, and indicate if changes were made. The Creative Commons Public Domain Dedication waiver (http://creativecommons.org/publicdomain/zero/1.0/) applies to the data made available in this article, unless otherwise stated. 


\section{Background}

Cemento-osseous dysplasia (COD) is an uncommon fibro-osseous lesion of the jaws with a periodontal ligament origin [1-3], which - in its early stage - can mimic a periapical lesion [4]. COD is a non-neoplastic, radiolucent and/or radiopaque, non-encapsulated, cellularfibrotic lesion with calcified structures - such as irregular osseous trabeculae and cementoid mass - affecting the tooth-bearing area and the cancellous part of the jaws [5] associated with vital teeth usually without any clinical sign or complaint [6]. This is the reason why many cases are diagnosed accidentally during radiographic examinations which were ordered for other reasons. The disease predominantly affects middle-aged Asian and African females [7, 8], and is often only recognized in an advanced stage. It is usually not prone to growth (except some cases of the florid form) and in many cases, the lesion closes spontaneously [9]. In the last, 4th edition of the WHO Classification of Head and Neck Tumours [10], cemento-osseous dysplasia was classified in the fibro- and chondro-osseous lesions, in the group of "benign odontogenic tumours and allied lesions". In the 2005 edition [11] it is also defined as a member of benign fibro-osseous dysplasias, but the term was changed to osseous-dysplasia without the prefix "cemento", stemming from the consideration that the cementum and the bone tissue can only be distinguished from each other by their origin and their respective relationship to the root $[4,12]$. Simply put, the mineralized concentric formation inside the lesion is referred to as cementum, while the long-shaped one as bone trabecula $[3,6]$. Though as in the case of the cemento-ossifying fibroma, the 4th edition of the WHO Classification reverts to the term "cemento-osseous dysplasia" with the purpose of highlighting the odontogenic origin of COD, emanating from the undifferentiated fibroblasts of the periodontal ligaments. COD has been recently divided into three subtypes based on the anatomical location: periapical, focal and florid. The occurrence of the familial type is very rare and according to the current classification, it only presents as the florid type [2]. COD can affect only one or several quadrants, both in the mandible and the maxilla.

\section{Case presentation}

\section{The first patient}

A 50-year-old Caucasian female visited the regional dental office due to uncertain pain in the right mandibular region. No significant diseases were mentioned in the anamnesis. The patient was diagnosed with gingivitis, and treated with a non-steroid anti-inflammatory drug and oral rinsing with chlorhexidine. The condition of the patient did not improve significantly. When she returned a week later, she had no alleviation, but labial paresthesia on the right side of her lip. As the cause of the new symptom remained unidentified, she was referred to the oral surgery group. Initially, the paraesthesia affected only the right side of the lower lip, but by this time it had spread to the skin of the right side of her chin. The oral surgeons decided to extract the lower right wisdom tooth, but the labial paraesthesia still persisted. Since there was an extended composite restoration in the lower right first molar, and translucency around its apical region was visible, the lesion was diagnosed as periapical granuloma. The lesion on tooth 46 was not close to the mandibular canal; this can be seen on Fig. 1. Therefore, root canal treatment was carried out, despite the uncertain result of the percussion, palpation and sensibility test. The transparent lesion around the apex and the negative sensibility test lead to the decision of endodontic treatment. Diagnosis was made by a dentist who is not a staff member of our Department. The treatment revealed that the pulp was vital. Our oral diagnostic team examined the patient, and we diagnosed the case as cemento-osseous dysplasia based on the panoramic radiograph showing apical translucency lesions around the anterior teeth with vital pulp (Fig. 1). The paraesthesia has been continually present ever since. Because the lesion around the lower right canine had already involved the cortical bone by CBCT scan (Fig. 2), a root canal filling and removal of the apical lesion were performed. On the CBCT image the involvement of the entire periapical region can be seen including the lingual region and the buccal cortical (Fig. 2). It became clear during the planning of the surgery that the blood vessels and nerves of tooth 43 would be injured.

The root canal treatment was carried out on the lower right canine in a single-visit treatment using local anaesthaesia. We explored the entire root canal length using a size \#15 hand instrument. The working length was determined using an apex locator, (Woodpex III, Guilin,China), then the length was also confirmed with radiographic imaging. Following the length determination, the root canal

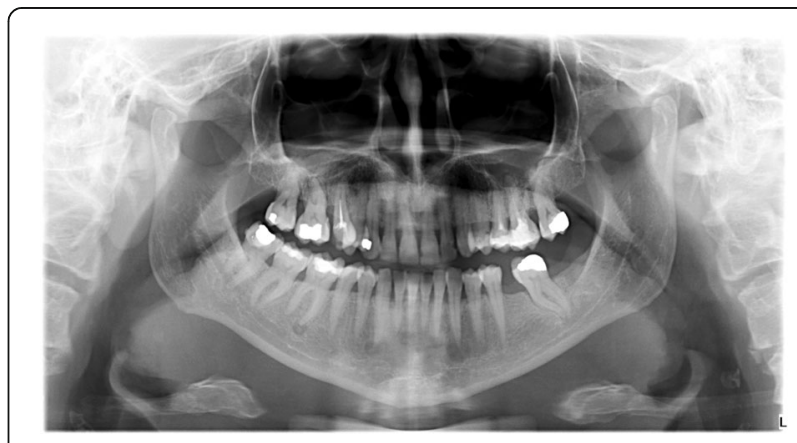

Fig. 1 Panoramic radiograph of the first patient. Radiolucent lesions in the mandible mimicking periapical endodontic lesions around vital teeth in the canine and the right molar region 


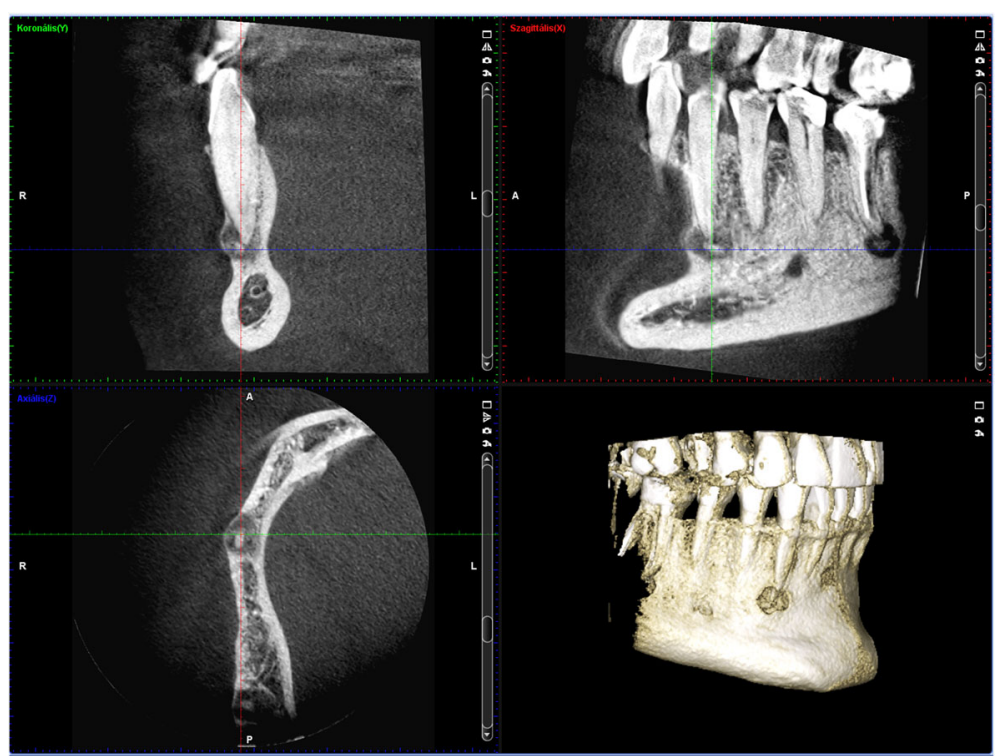

Fig. 2 The lesion on the lower right canine extruded and extremely thinned the buccal cortical bone on CBCT sections of patient 1

was shaped using Wave One (Densply Maillefer, York, USA) rotary instrument. The root canal was obturated using guttapercha and AH Plus sealer (Dentsply DeTrey $\mathrm{GmbH}$, Konstanz, Germany) with lateral condensation technique. Glass ionomer cement (Fuji IX GP, GC Co., Tokyo, Japan) was then applied to seal off the access cavity, while the permanent restoration was done.

We prepared an intraoral mucoperiostal flap using an L-shaped incision and the surgery was carried out by using a surgical microscope (Aspheron, Schmidt and Bender Hungaria, Budapest, Hungary).

We opened up the buccal cortical bone using a surgical bur, thereafter we removed the lesion surrounding the apex of the root. The root apex was resected and a retrograde root canal filling was placed using mineral trioxid aggregate (MTA+, Cerkamed, Stalowa Wola, Poland).

The area of the lesion was augmented using gentamicin impregnated BoneAlbumin (OrthoSera Dental Zrt.,

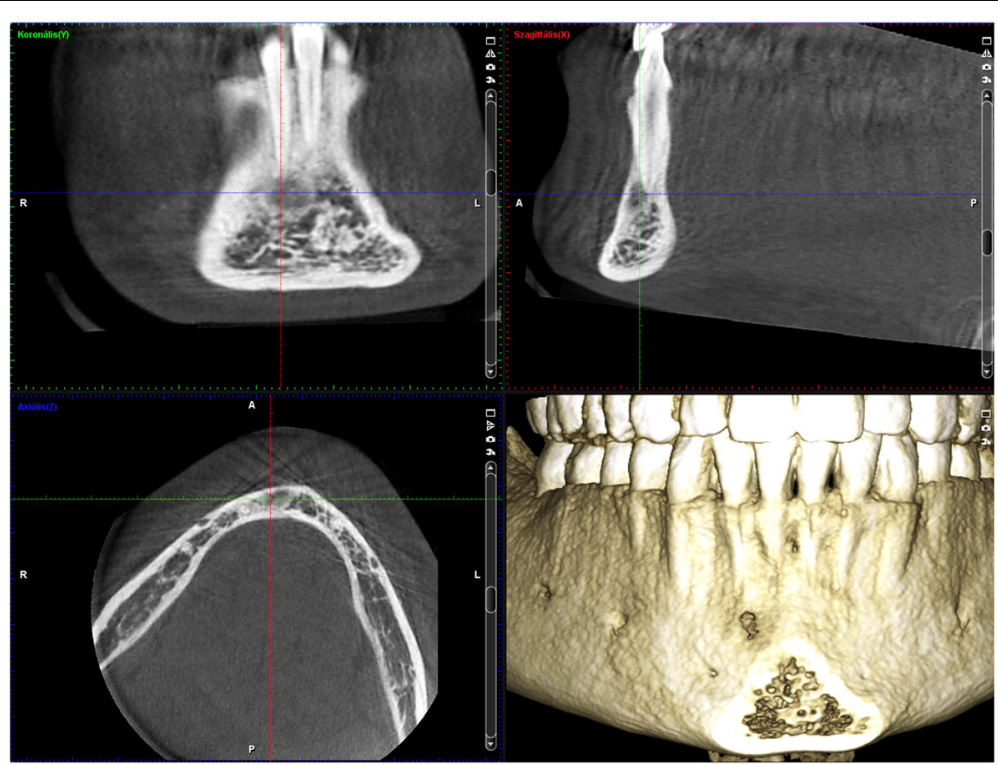

Fig. 3 Early radiolucent lesion without radiopaque area were revealed around the root apex of 42 tooth on CBCT sections of patient 2. The inner surface of the buccal cortical begun to be eroded by the lesion. This is obvious when one compares this with the contralateral side 
Gyor, Hungary). We seeked to lower the chance of osteomyelitis occuring with the use of gentamicin. Sutures were then carefully placed to achieve tensionfree closure of the flap for optimal healing.

\section{The second patient}

The 19-year-old Caucasian woman is the daughter of the first patient. CBCT showed a radiolucent lesion around the root of the lower right incisor (Fig. 3). She is presently asymptomatic after a one-year follow-up, but she occasionally felt tension and moderate pain in the right side of the mandibular region eradiating to her ear, approximately 2 years earlier. The symptoms had no dental background, and ceased gradually.

\section{Clinical examination}

Pulp tests and periapical pathology diagnoses were made by authors on teeth $33,32,41,43$ with the use of percussion, palpation and sensibility test.

\section{Radiological examination}

Panoramic radiograph and $\mathrm{CBCT}$ scans of the first patient showed radiolucent lesions located in the periapical bone, specifically on lower incisors and canines - in the premandibular, and in the right molar region of the mandible with radiopaque parts showing the lesions inside (Figs. 1 and 2). The affected teeth were asymptomatic, $\mathrm{CBCT}$ proved the presence of apical pathology. The buccal cortical involvement was discovered with the aid of CBCT, which also affected the treatment plan because 2D imaging methods provide no information on bucco-lingual dimension. Prior to the surgical intervention the use of CBCT was motivated by the fact that only $3 \mathrm{D}$ imaging could ascertain the precise shape, location and involvement of surrounding anatomical landmarks of the lesion.

Figure 4 shows the postoperative condition (Fig. 4). At the 6-months periapical follow-up, the bone healing is being processed (Fig. 5). 18 months later it can be seen on the CT image that the buccal cortical bone surrounding the root of tooth 43 has regenerated (Fig. 6). In the case of the second patient, there were no notable changes in the radiographic status 1 year later, and she has no complaint at present.

\section{Histopathology}

The removed tissue pieces contained both connective tissue and calcified areas. In the calcified area, beside the irregular trabecular - lamellar bony formations, oval and globular cementum-like structures were present. In the non-calcified area connective tissue was found and connective tissue filled out the centre of another bony sample as well. No elements or remnants of a capsule were visible. The histological diagnosis was cemento-osseous dysplasia (Fig. 7).

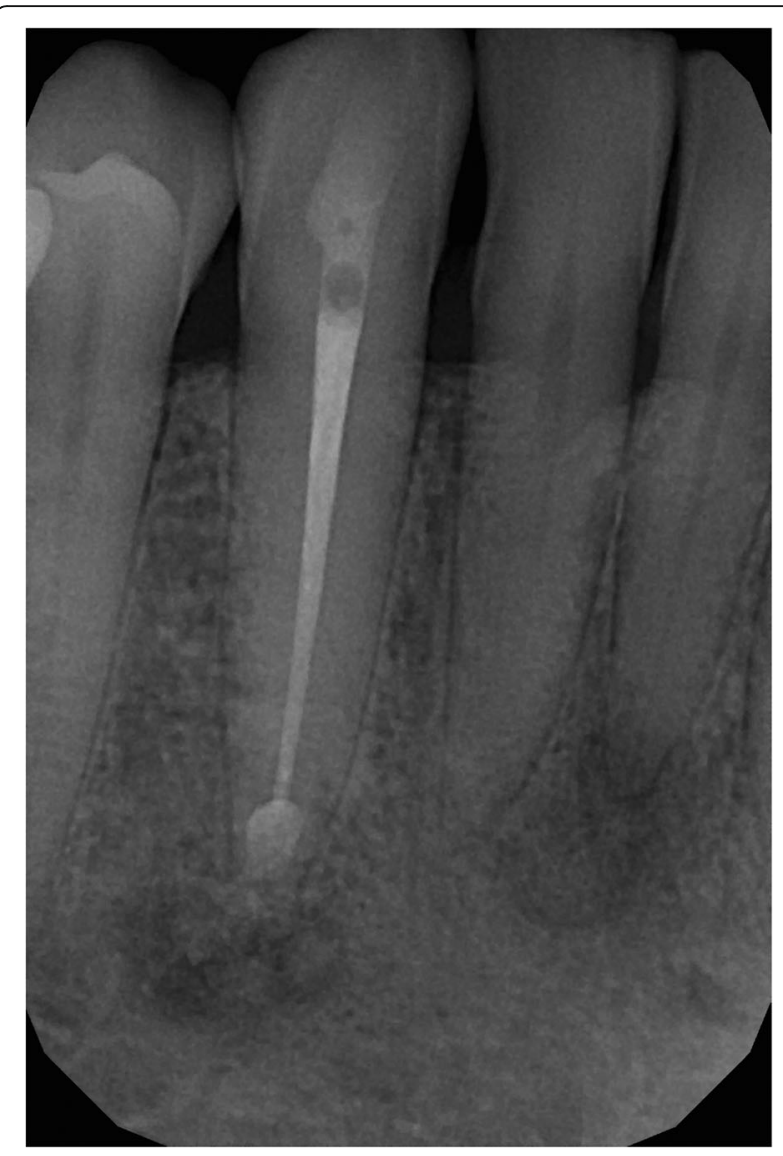

Fig. 4 On the apex of the first patient's lower canine a mixed (opaque and transparent) lesion is visible. Opaque focuses refer to remnants of the implanted bone graft material

\section{Discussion and conclusions}

COD is a sporadically occurring benign lesion in the tooth-bearing areas characterized by fibrosus dysplasia [6] .Familial occurrence of the disease is very rare [13], its characteristics differ from non-hereditary cases by its occurrence also in younger ages [14], and is not connected to the female gender and black skin colour. All familial cases are grouped in the florid group, and shows autosomal familial inheritance [2, 15-17].

The condition is usually asymptomatic, discovered in routine panoramic radiographs, and, in most cases, no treatment is necessary. Apart from certain cases of the florid form, lesions have limited growth potential. The progress of the disease can be divided into three stages: osteolytic, mixed and matured osteogenic [4].

In radiologic imaging COD can easily be confused with inflammatory periapical processes. The typical appearance is a mineralized area visible within the radiolucent lesion. The lesion usually goes through a maturation process, which results in an increasing number of radiopaque areas, until finally a no longer growing radiopaque area with a lobular, ginger root-like 


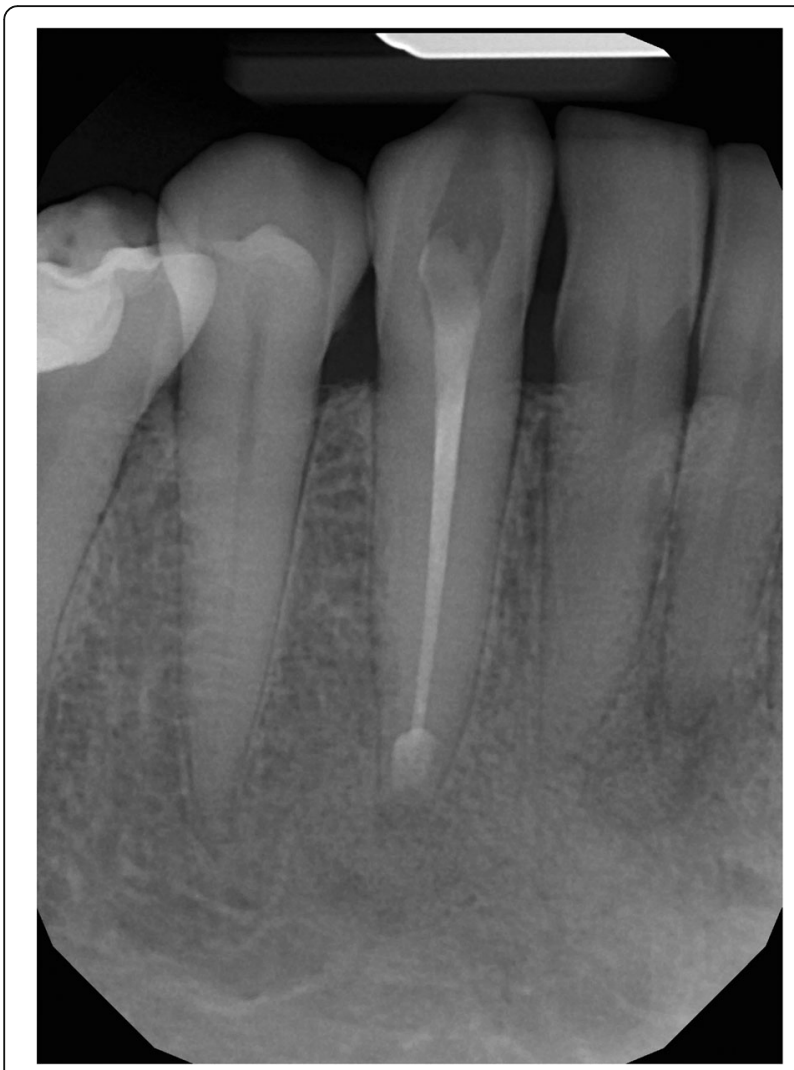

Fig. 5 At the 6-month follow-up of the first patient, a homogenous area is visible inside the lesion with a thin radiolucent margin

appearance remains. In the mixed and densest stage, a radiolucent border separates it from the surrounding healthy bone $[9,18]$.

Histologically, all forms of COD have similar appearance; the lesion is not surrounded by a capsule, and, in the early, osteolytic stage it consists of fibrotic tissue rich in cells and vessels, from which cement-like deposits are

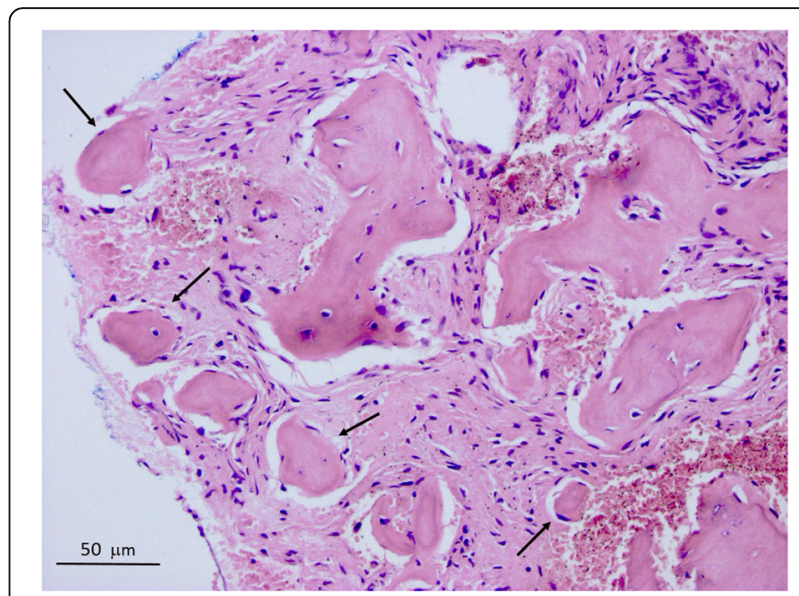

Fig. 7 Histological image of the COD lesion of the first patient. Acellular fragments of cementum-like substances (arrows) in loose fibro-collagenous stroma. Unmarked lamellar bony formations show no osteoclastic activity. $(H E, \times 400)$

absent. As the maturation progresses over time, cementlike formations and irregular trabeculae appear. In the final osteosclerotic stage, these structures connect to each other, and few cellular elements are visible [10, 19].

Differential diagnosis should be made between COD and the different forms of periodontitis in order to avoid unnecessary endodontic interventions [4, 20]. In the present case, the primary false diagnosis of the apical translucency around the lower right first molar resulted in an unnecessary root canal treatment, since the pulp was vital. Thereafter, the oral diagnostic staff was involved in defining the adequate diagnosis in order to prevent further unnecessary endodontic treatments of the anterior teeth. The present case raises the question of how many apical transparencies are misdiagnosed in general. Several cases were reported [21, 22] on

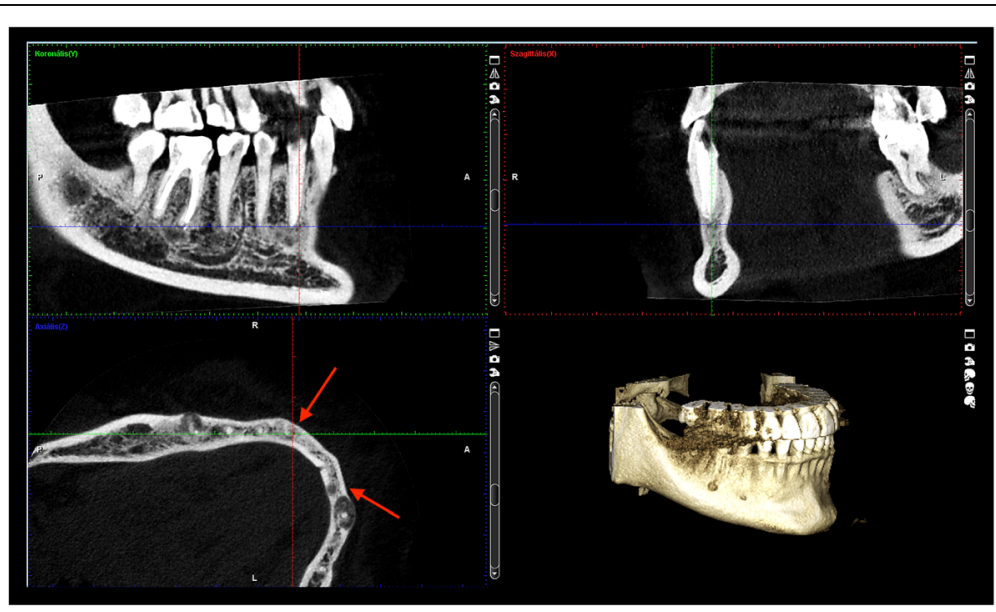

Fig. 6 Eighteen months follow up CBCT image of patient 1. Buccal cortical of lower right canine has regenerated. Swelling of this area disappeared. Periapical new bone showing lacunas and wider trabeculas filling the previous volume of pathology 
inadequate treatment with COD, however, these studies do not provide numbers or proportions of misdiagnosis.

Distinguishing from Paget's disease may also cause problems in the early stage. The microscopic image of Paget's shows the characteristics of fibro-osseous dysplasia [23]. COD may also be confused with fibrosus dysplasia - the distinction may be based on the differences of the mineralized matrix, the significant face asymmetry and the developing malocclusion. COD has recently been differentiated from cemento-ossifying fibroma, where the histological image is similar, but the lesion is encapsulated and clearly separated from the surrounding healthy bone tissue. The option of cementoblastoma also occurs - it always connects to the root apex and is also a tumour [24]. In the case of chronic sclerosing osteomyelitis, differentiation may be made based on the lack of inflammatory signs $[19,25,26]$.

Surgical treatment is limited for patients with severe clinical symptoms including pain and paraesthesia, those with expansive growth of the lesion, and candidates for dental implantation at the affected area $[8,19]$.

Terminology of COD has varied over the years. These changes depend on whether the focus is on the origin or the clinical presentation. In the latest WHO classification [10] three clinical forms of cemento-osseous dysplasia are distinguished:

\section{Periapical cemento-osseous dysplasia}

It connects to the root apex region of the premandibular teeth, affects one or more teeth and is usually asymptomatic. It often leads to a false diagnosis, which may cause unnecessary intervention $[4,27]$.

\section{Focal cemento-osseous dysplasia}

It is linked to one tooth, appears in the molar region in the tooth-bearing or edentulous area [6].

\section{Florid cemento-osseous dysplasia}

The term was introduced by Melrose [9] who described the lesion in 1976. Authors agree that it is inherited as autosomal dominant trait [16]. It can occur in any quadrant of the maxilla and mandible as a multifocal multiquadrant lesion, it is often expansive and may rarely cause the deformation of the jaw bone. All types of COD but most often the florid form can cause changes in the blood supply of the bone: therefore, it increases the risk of infections (e.g. chronical osteomyelitis) in connection with surgical interventions - tooth removal in the affected area, implantation and biopsy - and may lead to the development of chronic osteomyelitis [28, 29]. Most often, the existence and expansion of this form cause pain. Paraesthesia has also been reported [2], which was the leading symptom of our first patient.
In the previous publication of the WHO [30] familial gigantiform cementoma is mentioned as the other expansive form of osseous dysplasia, which also shows autosomal dominant inheritance [17]. This type occurs in young people and causes significant swelling of the mandible [31]. In the $2017 \mathrm{WHO}$ publication however, it is mentioned as an individual disease [10].

Just like some authors, such as Noffke places emphasis on the fact that the nomenclature should reflect the real nature of the lesion and recommends a different classification that takes both the clinical and biological characteristics into account. According to Noffke's experience, the epicentre of all lesions was in the periapical region, but none of them was related to the surface of the tooth root: therefore, she suggests that the word "cementum" should be omitted. In her opinion, the term "periapical cemento-osseous dysplasia" is also unnecessary, as the other subgroups also originate from the periapical region. Based on the growth potential she has proposed the following classification: expansive and non-expansive, and within the non-expansive group a distinction between florid, focal and anterior mandibular form should be made. Finally, the expansive osseous dysplasia should be divided into familial and non-familial groups [32, 33].

The importance of our case is that it is a rare hereditary form of COD. Two regions were affected in the mother, the anterior and the right posterior regions. The diagnosis of COD was confirmed by histology. Based on the clinical characteristics, the case was classified as florid COD. However, if we examine only the daughter, then the diagnosis would be periapical COD, because the pathology developed in the anterior region only. As familial inheritance was described associated only with the florid form, we must classify the second patient as belonging to the florid group according to the present classification. This inconsistency raised the possibility that the periapical and focal form could in fact be the initial or unifocal manifestation of the florid COD [26, $34,35]$. Neither the periapical nor the focal version turns necessarily in to the florid, multiplex disease: moreover, these can recover at any stage. To date no histological differences have been reported distinguishing these forms, and all the COD variants have the same microscopic features $[10,21]$. In the case of the daughter, a long-term follow-up will show whether further lesions will develop in other regions or not, which would mean that one COD form could turn into another. We agree with Summerlin who pointed out the possibility in his study that the single or initial condition may develop into the florid form [26]. For this reason, we think that the new classification proposed by Noffke is well established and more helpful for build-up treatment protocols: Raubenheimer, Noffke: "Dividing the OD's in nonexpansive- (conventional, with no- or minimal expansion 
and generally occurring in patients above the 3th decade of life) and expansive subtypes. This division provides clear guidelines on management, as the approach to the treatment of the expansive group is surgery and the nonexpansive types require no treatment unless infected" [34]. We might consider using only the focal term instead of the focal and periapical because "both are the same entity with different locations" [35, 36]. It is based on the fact that the origin of all subtypes of COD is the same - the cells of periodontal ligaments [1-3], so we have to divide the different forms not on the basis of the anatomical location but the clinical and genetic features. The early stage and familial appearance of COD cases presented in this study serve as an example that the different forms of the recent classification cannot be used strictly.

Cemento-osseous dysplasia is a rare benign fibroosseous disease of the jaws associated with vital teeth and generally no need for intervention, but follow-up is recommended. The differential diagnosis between the early stage of this fibro-osseous lesion and periapical inflammations and cysts is difficult to make but it is very important in order to avoid unnecessary endodontic treatments. On the other hand, we are faced with the problem that the current categories do not cover the early stage of a hereditary, possible florid form of cemento-osseous dysplasia. Familial occurrence of COD is very rare, and the previous reports found exclusively the florid subtype in all family members. In contrast, in the present report cemento-osseous dysplasia diagnosed in the mother and her daughter were of different subtypes. Our cases attract attention to the fact that the age range given in the literature is a mean calculated based on the time of recognition of the disease, and thus does not always follow the dinamics of the pathogenesis. Due to the fact that in the preliminary stages COD resembles periapical inflammation, therefore very few people diagnose it as COD. It is pertinent to control family members as well, and if a lesion is present make them aware of the implications.

\section{Abbreviations}

CBCT: Cone Beam Computed Tomography; COD: Cemento-osseous dysplasia; WHO: World Health Organization

\section{Acknowledgments}

Not applicable.

\section{Authors' contributions}

The corresponding author $A B$ examined and treated the patient, reviewed the literature and prepared the manuscript. AZ carried out and evaluated the hystopathology. CsDN supervised the patient treatment and the preparation of the manuscript, analysed and evaluated the CBCT radiographs. KCs carried out the surgical treatment. $A B$ contributed to patient treatment and to the preparation of the manuscript. All authors analysed the results, read and approved the final version of the manuscript.

\section{Funding}

No funding was obtained for this study.

Availability of data and materials

All data generated or analysed during this study are included in this published article [and its supplementary information files].

Ethics approval and consent to participate

Not applicable.

\section{Consent for publication}

We obtained written consents to publish this information of our study participants.

\section{Competing interests}

The authors declare that they have no competing interests.

\section{Author details}

'Department of Oral Diagnostics, Faculty of Dentistry, Semmelweis University, Szentkirályi u. 47, Budapest 1088, Hungary. ${ }^{2}$ Department of Pathology and Experimental Cancer Research, Faculty of Medicine, Semmelweis University, Üllői út 26, Budapest 1085, Hungary. ${ }^{3}$ Department of Medical Chemistry, Molecular Biology and Pathobiochemistry, Semmelweis University, Túzoltó u. 37-47, Budapest 1094, Hungary.

Received: 20 March 2019 Accepted: 1 July 2019

Published online: 10 July 2019

\section{References}

1. Hamner JE 3rd, Scofield HH, Cornyn J. Benign fibro-osseous jaw lesions of periodontal membrane origin. An analysis of 249 cases. Cancer. 1968;22(4) $861-78$

2. Kucukkurt S, Rzayev S, Baris E, Atac MS. Familial florid osseous dysplasia: a report with review of the literature. BMJ Case Reports. 2016;2016: bcr2015214162.

3. Waldron CA, Giansanti JS. Benign fibro-osseous lesions of the jaws: a clinical-radiologic-histologic review of sixty-five cases: part II. Benign fibroosseous lesions of periodontal ligament origin. Oral Surgery, Oral Medicine, Oral Pathology. 1973;35(3):340-50.

4. Daviet-Noual V, Ejeil AL, Gossiome C, Moreau N, Salmon B. Differentiating early stage florid osseous dysplasia from periapical endodontic lesions: a radiological-based diagnostic algorithm. BMC Oral Health. 2017;17(1):161.

5. Speight PM, Takata T. New tumour entities in the 4th edition of the World Health Organization classification of head and neck tumours: odontogenic and maxillofacial bone tumours. Virchows Arch. 2018:472(3):331-9.

6. Waldron CA. Fibro-osseous lesions of the jaws. J Oral Maxillofac Surg. 1993; 51(8):828-35.

7. MacDonald-Jankowski DS. Florid cemento-osseous dysplasia: a systematic review. Dentomaxillofac Radiol. 2003;32(3):141-9.

8. Owosho AA, Potluri A, Bilodeau EA. Osseous dysplasia (cemento-osseous dysplasia) of the jaw bones in western Pennsylvania patients: analysis of 35 cases. Pa Dent J (Harrisb). 2013;80(6):25-9.

9. Melrose RJ, Abrams AM, Mills BG. Florid osseous dysplasia. A clinicalpathologic study of thirty-four cases. Oral Surg Oral Med Oral Pathol. 1976; 41(1):62-82

10. El-Naggar AK, Chan JKC, Grandis JE, Takata T, Slootweg PJ: WHO classification of head and neck Tumours, 4th edn. Lyon; 2017.

11. Leon Barnes JWE, Reichart P, Sidransky D. Pathology and genetics of head and neck Tumours WHO classification of Tumours, 3rd Edition. Lyon: IARC; 2005.

12. Kramer IRH, Pindborg JJ, Shear M. The World Health Organization histological typing of odontogenic tumours. Introducing the second edition. Eur J Cancer B Oral Oncol. 1993:29(3):169-71.

13. Hatori M, Tachikawa T, Nagumo M. Familial florid Cemento-osseous dysplasia. Asian J Oral Maxillofac Surg. 2003;15(2):135-7.

14. Coleman H, Altini M, Kieser J, Nissenbaum M. Familial florid cementoosseous dysplasia--a case report and review of the literature. J Dent Assoc S Afr. 1996;51(12):766-70.

15. Sedano HO, Kuba R, Gorlin RJ. Autosomal dominant cemental dysplasia. Oral Surg Oral Med Oral Pathol. 1982;54(6):642-6. 
16. Toffanin A, Benetti R, Manconi R. Familial florid cemento-osseous dysplasia: a case report. J Oral Maxillofac Surg. 2000;58(12):1440-6.

17. Young SK, Markowitz NR, Sullivan S, Seale TW, Hirschi R. Familial gigantiform cementoma: classification and presentation of a large pedigree. Oral Surg Oral Med Oral Pathol. 1989;68(6):740-7.

18. Delai D, Bernardi A, Felippe GS, da Silveira Teixeira C, Felippe WT, Santos Felippe MC. Florid Cemento-osseous dysplasia: a case of misdiagnosis. J Endod. 2015;41(11):1923-6.

19. Yazicioglu D, TOAM UC, Omur D. Focal cemento-osseous dysplasia: a case report and literature review. Health. 2010;2(8):941-4.

20. Smith S, Patel K, Hoskinson AE. Periapical cemental dysplasia: a case of misdiagnosis. Br Dent J. 1998;185(3):122-3.

21. Drazic R, Minic AJ. Focal cemento-osseous dysplasia in the maxilla mimicking periapical granuloma. Oral Surg Oral Med Oral Pathol Oral Radiol Endod. 1999:88(1):87-9.

22. Huh JK, Shin SJ. Misdiagnosis of florid cemento-osseous dysplasia leading to unnecessary root canal treatment: a case report. Restor Dent Endod. 2013; 38(3):160-6.

23. Carrillo R, Morales A, Rodriguez-Peralto JL, Lizama J, Eslava J. Benign fibroosseous lesions in Paget's disease of the jaws. Oral Surg, Oral Med, Oral Pathol. 1991;71(5):588-92.

24. Slootweg PJ. Maxillofacial fibro-osseous lesions: classification and differential diagnosis. Semin Diagn Pathol. 1996;13(2):104-12.

25. Kato H, Nomura J, Matsumura Y, Yanase S, Ito T, Tagawa T. A case of florid osseous dysplasia associated with chronic sclerosing osteomyelitis requiring segmental resection. J Oral Maxillofa Surg Med Pathol. 2014;26(2):158-62.

26. Summerlin DJ, Tomich CE. Focal cemento-osseous dysplasia: a clinicopathologic study of 221 cases. Oral Surg, Oral Med, Oral Pathol. 1994; 78(5):611-20.

27. Senia ES, Sarao MS. Periapical cemento-osseous dysplasia: a case report with twelve-year follow-up and review of literature. Int Endod J. 2015;48(11): 1086-99.

28. Macdonald-Jankowski DS. Focal cemento-osseous dysplasia: a systematic review. Dentomaxillofac Radiol. 2008;37(6):350-60.

29. Saheb-Jamee M, Arbabi-Kalati F. Florid cement osseous dysplasia and chronic diffuse osteomyelitis in maxilla. ZJRMS. 2013;15(1):55-7.

30. Barnes L, Eveson JW, Reichart P, Sidransky D. Pathology and genetics of head and neck Tumours WHO classification of Tumours, 3 edn. Lyon: IARC; 2005.

31. Raubenheimer EJ, Noffke CE, Boy SC. Osseous dysplasia with gross jaw expansion: a review of 18 lesions. Head Neck Pathol. 2016;10(4):437-43.

32. Noffke CE, Raubenheimer EJ. Expansive osseous dysplasia: report of 9 lesions in an African population sample and a review of the literature. Oral Surg Oral Med Oral Pathol Oral Radiol Endod. 2011:111(2):e35-41.

33. Noffke CE, Raubenheimer EJ, MacDonald D. Fibro-osseous disease: harmonizing terminology with biology. Oral Surg Oral Med Oral Pathol Oral Radiol. 2012;114(3):388-92.

34. Raubenheimer $\mathrm{E}$, Noffke $\mathrm{C}$. Regarding the use of the term "cementum" in pathologic proliferations. Head Neck Pathol. 2018;12(4):629-30.

35. Singer S, Creanga A, Vyas R, Mupparapu M. Florid expansile cementoosseous dysplasia of the jaws: cone beam computed tomography study and review of the literature. J Orofac Sci. 2017;9(2):114-7.

36. Eversole R, Su L, ElMofty S. Benign fibro-osseous lesions of the craniofacial complex. A review. Head Neck Pathol. 2008;2(3):177-202.

\section{Publisher's Note}

Springer Nature remains neutral with regard to jurisdictional claims in published maps and institutional affiliations.

Ready to submit your research? Choose BMC and benefit from:

- fast, convenient online submission

- thorough peer review by experienced researchers in your field

- rapid publication on acceptance

- support for research data, including large and complex data types

- gold Open Access which fosters wider collaboration and increased citations

- maximum visibility for your research: over $100 \mathrm{M}$ website views per year

At $\mathrm{BMC}$, research is always in progress.

Learn more biomedcentral.com/submissions 